\title{
Kinematic primitives for walking and trotting gaits of a quadruped robot with compliant legs
}

\author{
Alexander T. Spröwitz *, Mostafa Ajallooeian, Alexandre Tuleu and Auke Jan ljspeert
}

Biorobotics Laboratory, École Polytechnique Fédérale de Lausanne, Lausanne, Switzerland

\section{Edited by:}

Martin Giese, University Clinic

Tuebingen/Hertie Institute, Germany

Reviewed by:

Luc Berthouze, University of

Sussex, UK

Gustavo A. Medrano-Cerda, Istituto

Italiano di Tecnologia, Italy

${ }^{*}$ Correspondence:

Alexander T. Spröwitz, Biorobotics

Laboratory, École Polytechnique

Fédérale de Lausanne, EPFL STIIB|

BIOROB, INN 237, Station 14,

CH-1015 Lausanne, Switzerland

e-mail: alexander.sproewitz@epfl.ch
In this work we research the role of body dynamics in the complexity of kinematic patterns in a quadruped robot with compliant legs. Two gait patterns, lateral sequence walk and trot, along with leg length control patterns of different complexity were implemented in a modular, feed-forward locomotion controller. The controller was tested on a small, quadruped robot with compliant, segmented leg design, and led to self-stable and self-stabilizing robot locomotion. In-air stepping and on-ground locomotion leg kinematics were recorded, and the number and shapes of motion primitives accounting for $95 \%$ of the variance of kinematic leg data were extracted. This revealed that kinematic patterns resulting from feed-forward control had a lower complexity (in-air stepping, 2-3 primitives) than kinematic patterns from on-ground locomotion (4 primitives), although both experiments applied identical motor patterns. The complexity of on-ground kinematic patterns had increased, through ground contact and mechanical entrainment. The complexity of observed kinematic on-ground data matches those reported from level-ground locomotion data of legged animals. Results indicate that a very low complexity of modular, rhythmic, feed-forward motor control is sufficient for level-ground locomotion in combination with passive compliant legged hardware.

Keywords: motion primitives, locomotion patterns, central pattern generator, quadruped robot, passive leg compliance, entrainment, principal component analysis, walk and trot

\section{INTRODUCTION}

The overlapping fields of functional leg anatomy, leg and body compliance, and neuro-control in legged locomotion are intensively researched. Results potentially allow insights into the structure and functionality of the nervous system of animals. Not surprisingly, roboticists have started researching bio-inspired, legged robot systems, both on the functional morphological level, and the controller level. Though intrinsically limited (Webb, 2001), robots are beginning to be used as proof-of-concept platforms (Raibert et al., 1984; Raibert, 1990; Full and Koditschek, 1999; Ijspeert et al., 2007; Umedachi et al., 2010; Zhou and Bi, 2012).

In this experimental work we present results by comparing basic patterns measured from kinematic leg data from in-air stepping movements of a suspended legged, compliant robot, and from on-ground locomotion of the same robot during lateral sequence walk and trot. We applied as measure the number of significant principal components (PCs) extracted from joint-angle data of the robot's compliant, multi-segment legs. We compared four parameter setups, altering the robot's gait control parameters between walk and trot, its speed, and the modules and complexity of its locomotor drive signals. For the robot hardware, special attention has been paid to the in-series and in-parallel leg compliance. The robot was designed such that its leg's compliance and cable-driven actuation were the medium of change of kinematic complexity between feed-forward-sent and observed kinematic joint patterns, through emerging mechanical entrainment during level-ground (flat ground) locomotion. During in-air leg movement, leg-joints were not exposed to gravitational or inertial forces acting on the robot body (they were only exposed to those acting on the leg's own light-weight segments). The robot's legs were hanging freely in-air, and replayed motion patterns represented the kinematic complexity of the feed-forward locomotor controller. During on-ground locomotion, leg-joints were compressed by gravitational forces acting on the robot body, and Newtonian dynamics acting at the robot's body eventually deflecting the robot's compliant limbs. Self-stable and self-stabilizing locomotion only emerged if appropriate feed-forward patterns were sent to the robot. In all other cases, the robot would stumble, fall, or move only very poorly, i.e., very slowly or even backwards.

Physical, biological leg compliance was found to function as energy recoil mechanism, allowing animals to re-use negative work, and reduce metabolic cost of locomotion (Alexander, 1984, 1990, 1991; Biewener and Blickhan, 1988). Sources of compliance were found both in muscles and muscle complexes (Witte et al., 1994; Labeit and Kolmerer, 1995; Wilson et al., 2003), and in tendons and aponeuroses (Alexander, 1977; Witte et al., 1997; Biewener, 1998; Gregersen et al., 1998; Lichtwark et al., 2007). If biological systems rely that strongly on in-series and in-parallel compliant "locomotion hardware," the nervous system producing motor control patterns has to be able to cope with leg compliance, and is required to send the corresponding control signals. Ivanenko et al. (2002) present experiments with walking humans, and in-air stepping with varying amounts of gravity, leading to no 
or limited sensory feedback through foot contact. They observe that during air-stepping "... motor patterns are transformed in simple harmonic angular motion of the lower limb segments associated with alternating activation of antagonist muscles" (cf. Ivanenko et al., 2002, p. 3087). Although they conclude on the role of peripheral sensory input, we are building in this work on the idea that the complexity of leg kinematics during locomotion can be increased through compliant, and purely mechanical components of the locomotor apparatus and its interactions with the ground. In turn, this could mean that the underlying layers of motor control do not need to send as complex control signals as one might have guessed from kinematic studies, while still achieving a sufficiently complex, adaptive kinematic output.

The spring-loaded inverted pendulum (SLIP) framework describes how an abstracted, energy conservative point-mass system can make use of a compliant, in-series elastic leg design to self-stably run (Blickhan, 1989; Seyfarth et al., 2002). In a SLIP simulation, minimal control effort is required to stabilize locomotion. Control of the leg's angle of attack is necessary, together with monitoring the system's hip apex height. A feed-forward SLIP controller will lead to self-stable locomotion patterns, also under the influence of small perturbations. Full and Koditschek (1999) explain the SLIP model as a "template." A biological or robotic legged system still requires an "anchor," to map the system mechanics to the template.

In this work, central pattern generator (CPG) control signals in feed-forward mode were implemented as high-gain position signals from the robot's RC-servo motors, actuating hip joints and leg length. Interpreting CPG output patterns as position signals presents an abstraction and simplification of animal motor control and actuation. In animals, motor control signals of the nervous system are interpreted by sets of antagonistic muscles and muscle groups to produce joint torques (Inman et al., 1952). Hence, animals can control their limbs in many modes, including "position-control" (muscle lengths leading to joint angles), but also with adjustable joint torques (Winter, 1983; Fischer and Blickhan, 2006).

With the help of a robotic tool like Cheetah-cub robot, this work's intention is to shed light on the interplay between rhythmic, modular feed-forward motor control, and the mechanical entrainment leading to stable gait patterns. Mechanical entrainment was a result from an in-series and in-parallel segmented, robotic, bio-inspired leg design, appropriate actuator control patterns, gravity and body dynamics, and ground contact during locomotion. Though not within the topic of quadruped legged walk and trot locomotion, entrainment in articulated robotic systems has been looked upon earlier; Lungarella and Berthouze (2002) designed a setup with a swinging humanoid robot, showing that physical entrainment led to a larger basin of attraction for the space of control parameters leading to stable swinging motions. Entrainment was also achieved at the presence of non-linear mechanical coupling of the humanoid to its environment (Berthouze and Lungarella, 2004). This is interesting because ground contact of Cheetah-cub robot also presents a non-linear perturbation (alternating leg swing and stance phase).

Here, the simplified case of a position-controlled system with serial compliance allows us to focus on few components, and dismiss the effects of additional control and hardware complexity (e.g., explicit control feedback loops, corresponding controller architecture, or torque control strategies). As mentioned earlier, animals feature components of (simple) in-series leg compliance (Alexander, 1984; Gregersen et al., 1998). This morphological feature works also well for robots. In-series compliance can lead to reduced impact forces, what consequently can reduce the control complexity (Meyer et al., 2006).

In the presented experiments, change of complexity between the directly commanded in-air leg kinematics and the onground locomotion leg kinematics emerged through the robot's compliant leg design and the interactions with the ground. At level-ground locomotion, periodic leg length shortening is caused (a) by signals of the robot's feed-forward controller and (b) by the robot-body's pitch, roll, vertical, and translational body movements (Figures 2-2-5). The inertia- and gravity-induced robot body and leg length movements present a major difference between Cheetah-cub robot and the controller applied in this work, and other feedback-controlled and body-stabilized quadruped robots (Raibert et al., 1986; Havoutis et al., 2013). If one would design a similar set of experiments, but sent feedforward signals to a high-gain position controlled robot with stiff leg design, its kinematic data from in-air running would (largely) match that of on-ground running experiments. Hardware springs and cable clutches are not the only way to achieve deviating, adaptive joint kinematics. Alternative setups can facilitate low-gain position controlled actuators, or more generally torque or force controllers (Buchli et al., 2009; Valenzuela and Kim, 2012). These setups, however, require explicit feedback control.

Kinematic leg patterns of Cheetah-cub robot were extracted by principal component analysis (PCA; Krzanowski, 1988; Jolliffe, 2002) on the normalized kinematic leg data, for both in-air leg movements, and on-ground locomotion. Applying PCA on kinematic leg data from recording of locomotion experiments, or corresponding electromyographic data of the participating muscles has become a common tool in biology, and neurobiology. Dominici et al. (2011) present in a comparative study basic patterns derived from electromyographic (EMG) data of stepping human neonates, toddlers, pre-schoolers, and adults. The authors compare these results to data of neonatal rats, and adult quadruped animals such as cats and monkeys. They report that human neonate stepping and neonatal rat stepping can be represented by two basic patterns, and that human toddler locomotion activation patterns along with all adult quadruped animals share a similar pool of four basic patterns. However, toddler and human adult patterns show differences: “... the four patterns [of human adults] were accurately timed around the four critical events of the gait cycle ..." (cf. Dominici et al., 2011, p. 998). Basic patterns from toddler locomotion were less time structured. Dominici et al. (2011) conclude that the increase in patterns is caused by continuous learning, until adulthood. The similarity between toddler data, and basic patterns of adult quadrupedal animal locomotion provides a possible explanation as to where gait patterns in vertebrates originate: central pattern generators located in the spinal cord (Delcomyn, 1980; Grillner, 1985; Ijspeert, 2008). Moro et al. (2013a) extracted four basic patterns (kinematic motion primitives, $\mathrm{kMPs}$ ) from horses, for the three gaits 
walk, trot, and gallop (kMPs account for 93\% and 97\% of the kinematic data). Moro et al. (2013b) found five kMPs accounting for almost all variance of human walking and running kinematics. Ivanenko et al. (2004) reported five basic muscle activation patterns accounting for almost all variance of muscle activation, during human walking. Koditschek et al. (2004) reported observations on retrieving basic patterns from running cockroaches. Despite the animal's very high number of degrees of freedom-a cockroach is six legged and has multi-segment legs_ “... a single component represen[ted] over $80 \%$ of the variation ..." (cf. Koditschek et al., 2004, p. 256), for very fast running. The authors report that almost all variations were captured by three basic components, leading to the conclusion that a very simple neural controller was likely responsible for the motor control of this insect. The above findings from insects and mammals suggest that basic locomotion on level-ground requires three to five basic patterns, and possibly fewer for very fast locomotion. It is intriguing to be able to hypothesize and through robot hardware implementation and experimentation, test the interplay between locomotion controller, robot morphology, and locomotion patterns. Although direct conclusions can only be drawn for the artificial, robotic system, similar designs of both systems and similar results for the task of locomotion can provide insights into animal locomotion control, and how neuro-control is interacting with bio-mechanical components.

We recorded joint-angle leg kinematics of our quadruped robot, for two situations: in-air trotting movements, versus robot locomotion on-ground (Figure 1). With its legs swinging inair and without contacting the floor, the kinematics of the robot's low-inertia leg-joints followed the commanded patterns. Once Cheetah-cub robot is placed on-ground to walk or trot, the interplay between ground contact, in-series leg compliance and spring deflection, and body movements alters the complexity of its leg kinematics. In all experiments documented, motor control patterns where sent feed-forward. Hence, the observed changes between in-air and on-ground leg kinematics were caused by ground contact and mechanical entrainment.

The experiments of this work are intended to inform on the potential interplay of a compliant, legged system, such as found in legged animals or robots, and its motor control. In animals, the interplay of the brain, spinal cord, peripheral nervous system (PNS), morphology, and intrinsic, mechanical properties (springiness, damping, inertial moments, lengths, speed and force properties) plays a key role for its locomotion capabilities. Studies like Ivanenko et al. (2002) or Hägglund et al. (2013) show that understanding the role, structure, and interplay of locomotor components in animals is difficult. With a largely simplified robot "hardware model" we can focus on the interplay of only a few components. Specifically, this robot features only these locomotor components: a feed-forward, oscillatory motor controller (we implemented a central pattern generator, but any other controller with similar features could be applied), and the mechanically compliant, bio-inspired leg structure. Cheetah-cub robot is stripped off any task-level control feedback, the motor control CPG is purely running in feed-forward mode. Self-stable and self-stabilizing locomotion was a product of appropriate motor control patterns (derived through systematic testing, see Spröwitz et al., 2013), the robot's compliant leg design, and the mechanical entrainment of these components, through ground contact during on-ground locomotion.

Establishing a reduced experimental setup, without any tasklevel feedback from the nervous system, is hard to achieve in live, locomoting animals. Possibilities for modulation of PNS pathways include preparations with drugs, lesions (see for example Grillner and Zangger, 1979), or more recently introduced methods like light-evoked activation and deactivation of spinal cord and PNS components (Daou et al., 2013; Hägglund et al., 2013). The vast number of publications in the field shows the complexity of locomotion generation and control in animals. From this perspective, the use of a legged robot with a programmable motor controller and dedicated hardware presents a diametrical, bottom-up approach to analyze the interplay of only its featured, however, much simpler locomotor components.

As our first hypothesis (H1-1) we expect four to five basic patterns accounting for $95 \%$ of the variance of kinematic leg data, at low-speed and mid-speed robot locomotion, as observed from legged animals (Ivanenko et al., 2004; Dominici et al., 2011; Moro et al., 2013a,b). Further, Koditschek et al. (2004) had reported a decreasing number of motion primitives at high locomotion speed, for a simpler biological legged systems with leg compliance. Hence, we expect a similar trend: a decreasing number of on-ground PCs with increasing robot speed (H1-2).

In addition, we looked at the number of in-air stepping PCs, versus the number of on-ground locomotion PCs. The interaction of ground contact, feed-forward controlled compliant legs, and the naturally emerging pitch and roll body movements produced a self-stable walk or trot gait (Spröwitz et al., 2013). This richer, additional pattern through mechanical entrainment should be visible as a higher number of observed basic principal components for on-ground locomotion, compared to non-contact, in-air stepping. In-air stepping presents virtual locomotion patterns with rigid, non-compliant legs. No ground contact deflects the serial leg springs out of their slack length, and recorded complexity of in-air kinematic data represents effectively the complexity of Cheetah-cub's feed-forward controller. As our second hypothesis we expect a higher number of basic patterns for on-ground locomotion, compared to in-air stepping (H2).

This paper is organized as follows: in section 2 we give a short overview of the robot's locomotion controller and hardware. We provide details of data recording and processing, and of the extraction of basic primitives from the recorded kinematic data. In section 3 we present the results from the four proposed experiments for walk and trot locomotion, in-air and on-ground. In section 4 we discuss results and their implications for biological and robotic systems. Finally, we conclude the paper.

\section{MATERIALS AND METHODS}

The first part of this section covers a brief description of the robot's hardware. For a more throughout description please refer to (Spröwitz et al., 2013). Next, this section provides information about the experimental tools and setup, and details about the applied principal component analysis. Cheetah-cub robot's gait controller based on a central pattern generator (CPG in feed-forward mode) is explained. Videos of Cheetah-cub robot running can be found at 

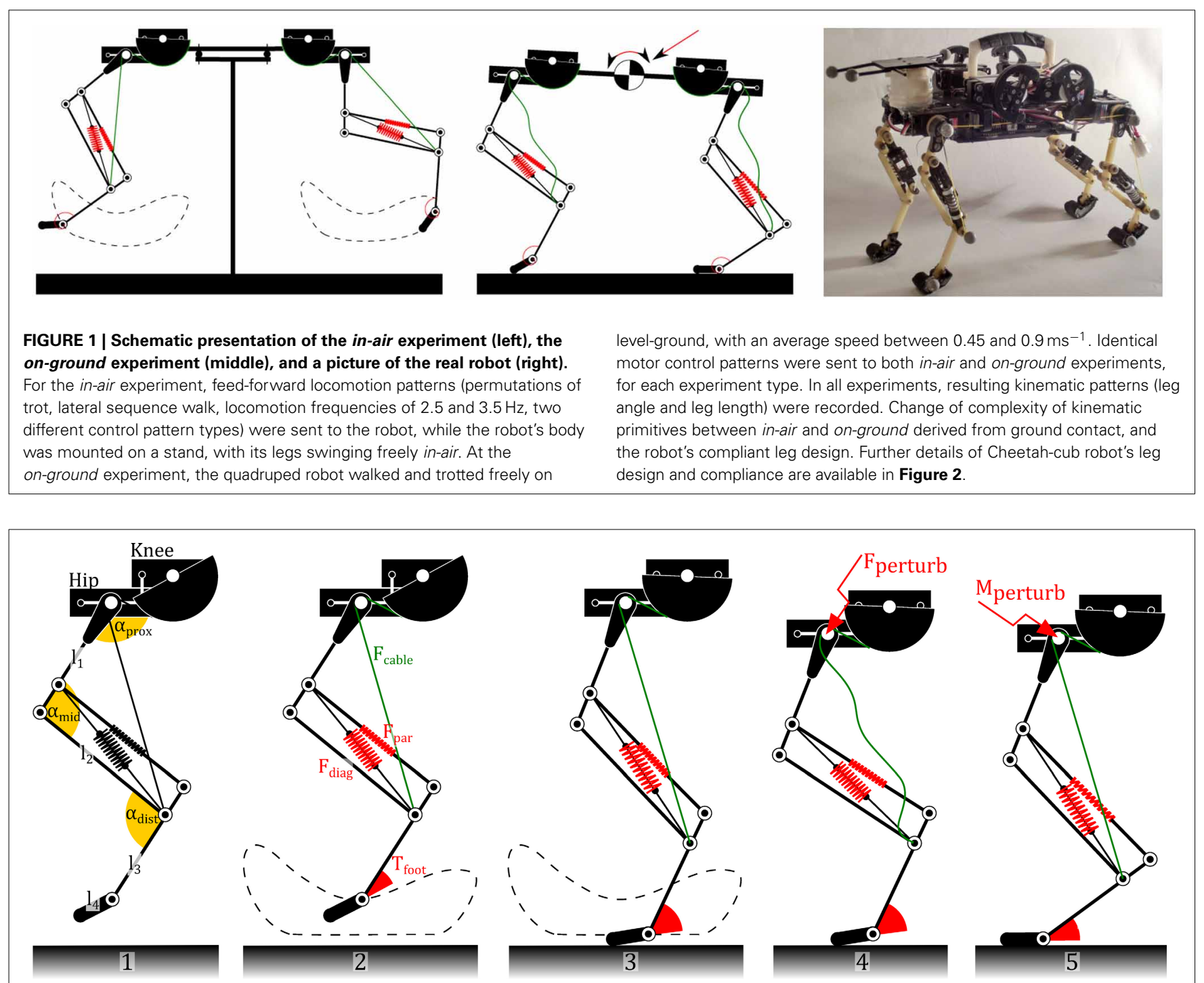

FIGURE 2 | Cheetah-cub leg mechanism, and leg compliance. A single leg is shown abstracted, detailed leg segment ratios are omitted for clarity, robot heading direction is to the left. (1) shows the three leg angles $\alpha_{\text {prox }}, \alpha_{\text {mid }}$, and $\alpha_{\text {dist }}$. Hip and knee RC servo motors are mounted proximally, the leg length actuation is transmitted by a cable mechanism. The pantograph structure was inspired by the work of Witte et al. (2003) and Fischer and Blickhan (2006).

(2) The foot segment describes a simplified foot-locus, showing the leg in mid-swing. For ground clearance, the knee motor shortens the leg by pulling on the cable mechanism (green, $F_{\text {cable }}$ ). $F_{\text {diag }}$ is the major, diagonal leg spring. Its force extends the pantograph leg, against gravitational and dynamic forces. (3) The leg during mid-stance. (4) In case of an external translational perturbation, the leg will be compressed passively. (5) If an external perturbation torque applies e.g., through body pitching, the leg linkage will transmit it into a deflection of the parallel spring, not of the diagonal spring. http://biorob2.epfl.ch/utils/movieplayer.php?id=209 and http:// biorob2.epfl.ch/utils/movieplayer.php?id=207.

\subsection{OUADRUPED ROBOT HARDWARE}

Cheetah-cub robot's leg design is based on a mammalian, animalinspired pantograph mechanism (Witte et al., 2003; Fischer and Blickhan, 2006). An automatic, cable-based clutch mechanism, proximal actuation, and a compliant foot joint enhanced the original, bio-inspired hardware blueprint (Spröwitz et al., 2013). Each robot-leg was individually controlled by two RC servo motors. The leg length (knee) actuator actively flexed the leg via a cable mechanism, antagonistic to the diagonal leg spring (Figure 2-2).
The cable mechanism also works as an automatic decoupling mechanism. It goes slack if external forces are applied to the leg (Figure 2-4). The robot's proximal actuator was directly mounted between body and leg. It protracted and retracted front and hind legs.

The robot's body was implemented as a stiff plate, only legs provided compliance. Three leg springs are acting in this leg design, under different load conditions (Figure 2): $F_{\text {diag }}$ is the inparallel spring to the cable actuation, and provides anti-gravity support. $F_{\text {par }}$ is the spring replacing one of the struts of the linkage mechanism. Under tension it provides an in-series leg elasticity. The third spring is located in the most distal leg joint. It 
is a helical spring and provides serial foot torque. In sum, this presents a very compliant leg design with a very low leg stiffness, in comparison with biological systems. The linearized vertical leg stiffness of two in-parallel Cheetah-cub robot legs is about $0.25 \mathrm{kN} / \mathrm{m}$, for a static measurement with isolated legs. During fast trotting locomotion (Froude number speed of $\mathrm{FR}=1.0$ ), a leg stiffness of $F_{\text {vert }} / \Delta l=0.65 \mathrm{kN} / \mathrm{m}$ was recorded. Leg stiffness for running, quadruped animals of this body weight, but at faster speed, are documented to be almost twice as high $\left(k_{\text {leg }}=\right.$ $M^{0.67}=1.05 \mathrm{kN} / \mathrm{m}$, using the convention of treating all groundcontacting legs as one, Farley et al., 1993). Compared to a young cat of equal weight, Cheetah-cub robot exhibits a more crouched leg posture. This is generally associated with a lower overall leg stiffness through bigger effective lever arms.

In all experiments, the robot was tethered to a power supply through a long, light-weight power cable. CPG computation, RC servo motor control signal generation, and wireless communication were controlled from a single board computer, mounted on the robot's body.

\subsection{DATASET, EXTRACTION, AND EXPERIMENTAL SETUP}

The robot was controlled with four different sets of control parameters (Figure 4). The robot ran at two different gait patterns (lateral sequence walk, and trot), a speed range from mid-speed to higher-speed, and two different knee-control strategies. Thus, the robot exploited its body dynamics for different gait patterns, control complexities, and dynamical speed conditions: (a) Lateral sequence walk gait with a locomotion cycle frequency of $2.5 \mathrm{~Hz}$, with double-peak knee deflection (DP, Figure 3), at medium robot speed. (b) Trot gait with a locomotion cycle frequency of $3.5 \mathrm{~Hz}$, with DP knee actuation, at high robot speed. (c) Trot gait with a locomotion cycle frequency of $2.5 \mathrm{~Hz}$, with DP knee actuation, at medium robot speed. (d) Trot gait with a locomotion cycle frequency of $3.5 \mathrm{~Hz}$, with a single-peak knee actuator signal (SP), at higher [compared to (a) and (c)] robot speed. Single-peak and double-peak leg length control signals, and hip-joint control signals are plotted for one locomotion cycle in Figure 3. All robot runs were repeated 10 times, and between 30 and 60 stride cycles were extracted for each gait. Kinematic robot data was recorded with a motion capture (MOCAP) system, based on infrared reflective markers of $11 \mathrm{~mm}$ diameter. Twelve MOCAP cameras (Optitrack s250e, Naturalpoint, Inc., 2011) were mounted at $1.20 \mathrm{~m}$ and $2.30 \mathrm{~m}$ height, positioned in a large rectangular arena around the locomoting quadruped robot. Cameras observed a volume of $1.5 \mathrm{~m}$ width, $4 \mathrm{~m}$ length, and $0.5 \mathrm{~m}$ height. MOCAP data were captured at $f=250$ fps. Marker trajectories were processed and cleaned with Arena software (Naturalpoint, Inc., 2011). Unlabeled markers were labeled in Mokka (Barré and Armand, 2014). Data was loaded in Matlab (MATLAB, 2009, v. 7.9) with $b$-tk framework (Barré and Armand, 2014). All marker trajectories were low-pass filtered, with an $18 \mathrm{~Hz}$ cut-off frequency.

For in-air experiments, Cheetah-cub was mounted on a small stand in the center of the arena. Its legs were hanging freely in the air, and MOCAP cameras recorded leg kinematics. For on-ground experiments, Cheetah-cub ran the full length of the motion capture arena distance $(4 \mathrm{~m})$, without restraints. The robot was powered externally by a power tether. Cheetah-cub's design includes no explicit degree of freedom for changing direction, i.e., adduction or abduction. Before reaching its steady state and before recording was started, the power tether was, in a few cases, used to correct the robot's heading. During recording, the robot would walk or trot while the tether was carefully kept loose. The robot was started from the ground, data was recorded once it reached steady state. This happened typically after less than three locomotion cycles. Markers were attached on the robot's right side of fore and hind limb, on the proximal leg joints, midleg joints, and feet (Figures 1, 2). Using leg kinematics, angles of proximal $\left(\alpha_{\text {prox }}\right)$, middle $\left(\alpha_{\text {mid }}\right)$, and distal $\left(\alpha_{\text {dist }}\right)$ joints were calculated (Figure 2-1). As the robot leg's parallel spring and foot spring work in-series, deflection of the distal leg segment and the foot segment were combined for simplification into a single angle $\left(\alpha_{\text {distal }}\right)$. Recorded locomotion stride cycles were synchronized based on pi/ 2 crossing of hip angle of the virtual leg (hip to foot), at mid-swing. The end of each stride cycle was calculated from the inverse stride frequency. Finally, all cycles were divided into 100 samples per cycle, using Piecewise Cubic Hermite Interpolating Polynomial (Fritsch and Carlson, 1980).

\subsection{PRINCIPAL COMPONENT ANALYSIS, PCA}

Kinematic primitives describing the main components of the kinematic leg dataset can be computed by matrix factorization (Strang, 2003). We applied Principal Component Analysis (Krzanowski, 1988; Jolliffe, 2002) to implement matrix factorization (similar to Fod et al., 2002; Bizzi et al., 2008). The obtained data are represented with $\mathbf{X}_{n_{o}, n_{v}}$, with $n_{o}$ being the number of observations, i.e., the number of samples per cycle, and $n_{v}$ being the number of variables, i.e., the number of cycles. The dataset was first normalized $\tilde{\mathbf{X}}_{n_{o}, n_{v}}$, so each cycle had a zero mean and a standard deviation equal to one. The covariance matrix of the normalized dataset was then calculated, obtaining $\Sigma_{n_{v}, n_{v}}$ :

$$
\Sigma=\frac{1}{N-1} \sum_{i=1}^{N}\left(\tilde{X}_{i}-\bar{X}\right)^{T}\left(\tilde{X}_{i}-\bar{X}\right)
$$

where $\tilde{X}_{i}$ is the $i$-th observation in $\tilde{\mathbf{X}}$, and $\bar{X}$ is the mean observation. Principal components of the covariance matrix were extracted, obtaining the loading vectors $\mathbf{v}_{i}, i=1 . . \mathrm{min}$ $\left(n_{o}-1, n_{v}\right)$, and the respective eigenvalues $\lambda_{i}$, sorted in descending order. Typically a low number of principal components are sufficient to account for a big part of variance. If the first $n_{s}$ components account for a percentage variance (e.g., $n_{s}$ components account for $95 \%$ of the variance, for all results in this work), then $n_{s}$ primitives are obtained by projecting the normalized dataset on the most significant loading vectors $\mathbf{v}_{i}, i=1 . . n_{s}$ :

$$
\mathbf{p}_{i}=\tilde{\mathbf{X}} \mathbf{v}_{i}
$$

\subsection{LOCOMOTION CONTROL WITH CENTRAL PATTERN GENERATOR}

Central pattern generators (CPG) were successfully applied to generate locomotion patterns for legged and other robots (Fukuoka et al., 2003; Ijspeert, 2008; Spröwitz et al., 2008, 2013; Sato et al., 2011). We applied a CPG implemented 
as a network of coupled oscillators to rapidly and conveniently encode a feed-forward control signal with explicit, legged locomotion-relevant input parameters such as duty factor, hip amplitude, leg length, and locomotion frequency. Cheetah-cub is a RC-servo motor controlled quadruped robot. The CPG provides for smooth trajectory transition at gait initialization, because of damping terms in the CPG equations. The CPG controller was running feed-forward, i.e., it was streaming a position signal to the RC-servo motors, without incorporating external feedback. Cheetah-cub robot's CPG controller consisted of two modules, a hip controller, commanding the hip motor, and a knee controller, commanding the leg length through a proximally mounted knee motor. Two knee control strategies (single-peak SP, and double-peak DP) were implemented. An example locomotion cycle is provided in Figure 3. Top and center plots show a gait applying double peak knee-signals (DP). Top and bottom plots show a gait with a single-peak (SP) knee-signal. The hip signal (top plot) is identical for SP and DP gaits. We used previously derived CPG parameters for trot gait (Spröwitz et al., 2013). The hip-joint-driving CPG consisted of a network of four phase-coupled oscillators, each oscillator controlled one hip joint. The gait was switched from lateral sequence walk (cf. Hildebrand, 1989) to trot by setting the phase shift between hip oscillators accordingly (Righetti and Ijspeert, 2008). Knee oscillators of the corresponding knee were coupled serially to their hip oscillator. The range of speeds obtained for walk and trot is shown in Figure 4.

\section{RESULTS}

This section provides the results from four experiments, each for in-air stepping and on-ground locomotion of the robot. The number and shapes of basic components accounting for at least 95\% of the variance of the kinematic leg data are provided.

\subsection{DOUBLE-PEAK (DP) KNEE PATTERN, WALK, $F=2.5 \mathrm{HZ}$}

Figure 5 shows joint-angle data in-air and onground (Figures 5A,B), and the corresponding principal components (Figures 5C,D). Average robot speed for this experiment was $0.45 \mathrm{~ms}^{-1}$, around 2.3 body lengths per second. Figures 5C,D indicate that more than $97 \%$ of the in-air patterns can be presented by three principal components, and $96 \%$ of the on-ground joint-angles kinematics by four principal components. The first PC of in-air and on-ground represents almost $50 \%$ of the kinematic data, for both cases (Figure 9A).

\subsection{DOUBLE-PEAK KNEE PATTERN, TROT, $\boldsymbol{F}=\mathbf{3 . 5} \mathrm{HZ}$}

Average robot speed for the $3.5 \mathrm{~Hz}$ trot gait with double-peak knee actuation was the highest of all four experiment types $\left(0.9 \mathrm{~ms}^{-1}\right)$. Higher average robot speed is possible with higher motor coil voltage, up to $1.42 \mathrm{~ms}^{-1}(\mathrm{Fr}=1.3$, Spröwitz et al., 2013). However, the power consumption becomes so large that motors would break at longer experimentation, caused by coil overheating. A speed of $0.9 \mathrm{~ms}^{-1}$ was a compromise between fast robot locomotion, around 4.5 body lengths per second, and a repeatable, robust experimental setup. The results in Figure 6 show three basic patterns for in-air stepping (98\%), and four basic patterns for on-ground locomotion (97\%). Figure 9B shows

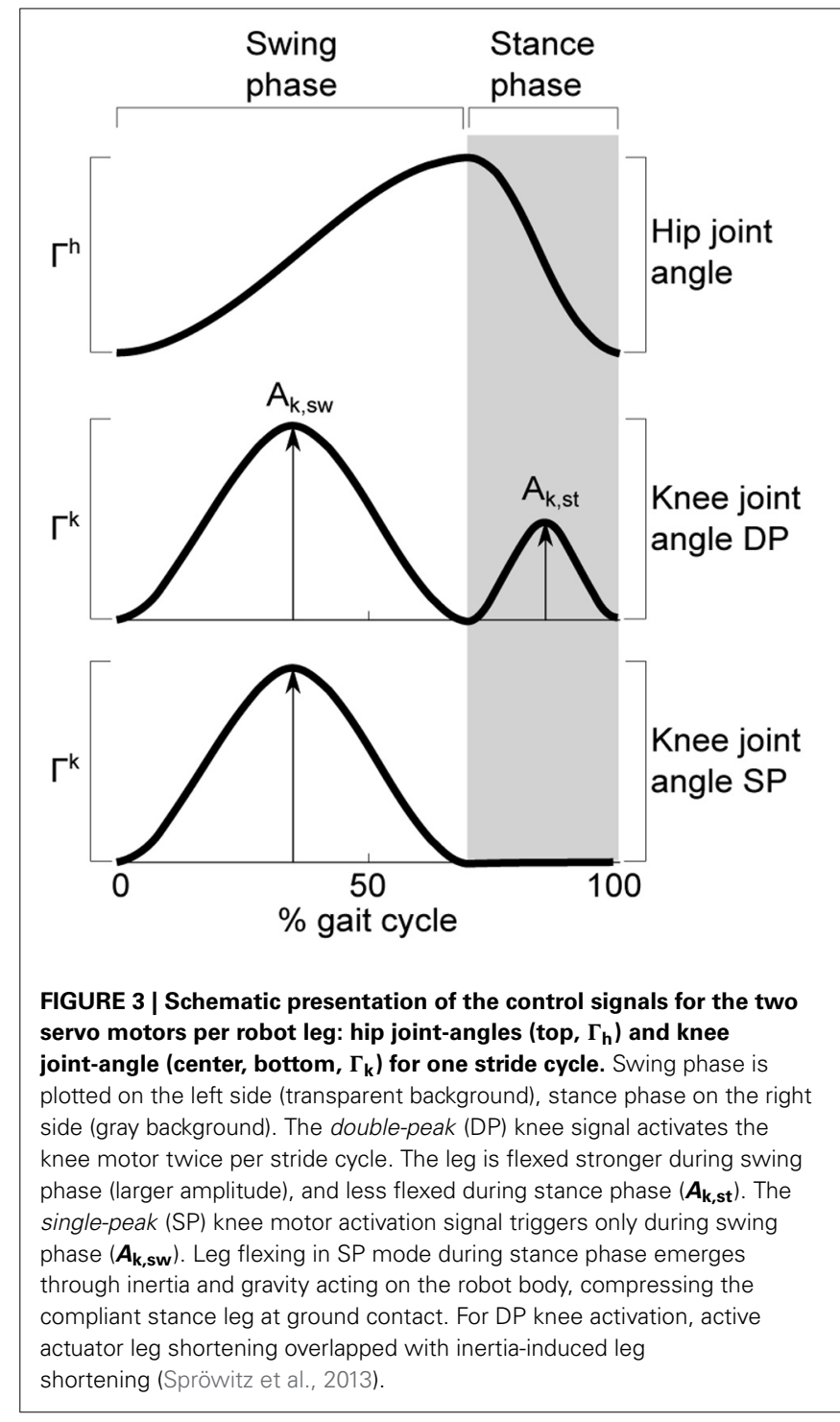

that the first in-air PC of this gait accounted for more than $60 \%$ of the variance of the kinematic data.

\subsection{DOUBLE-PEAK KNEE PATTERN, TROT, $F=2.5 \mathrm{HZ}$}

In this experiment the robot trotted at mid-speed level, at $v_{\mathrm{av}}=$ $0.55 \mathrm{~ms}^{-1}$. This was little more than the average speed of the robot for walk-gait experiment $\left(0.45 \mathrm{~ms}^{-1}\right)$, at the same gait frequency. Three basic patterns account for $98 \%$ of the variance of the in-air stepping data (Figures 7, 9C). Four basic components account for $97 \%$ of the variance of the on-ground locomotion joint-angle data.

\subsection{SINGLE-PEAK (SP) KNEE PATTERN, TROT GAIT, $\boldsymbol{F}=\mathbf{3 . 5} \mathrm{HZ}$}

Single-peak (SP) knee actuation based locomotion was only stable above a speed of $0.55 \mathrm{~ms}^{-1}$. The single activation burst of the knee actuator, effectively shortening leg length, is triggered during swing phase. This provides leg ground clearance to freely swing the leg and foot forward. Without leg length actuation during stance phase, leg shortening relies solely on inertia and gravity 
forces acting on the robot body compressing (flexing) the leg. This necessary amount of inertial energy explains the minimum required speed for SP experiments. Figure 8 shows two almost sine-shaped PCs for in-air stepping. Both PCs account for $98 \%$

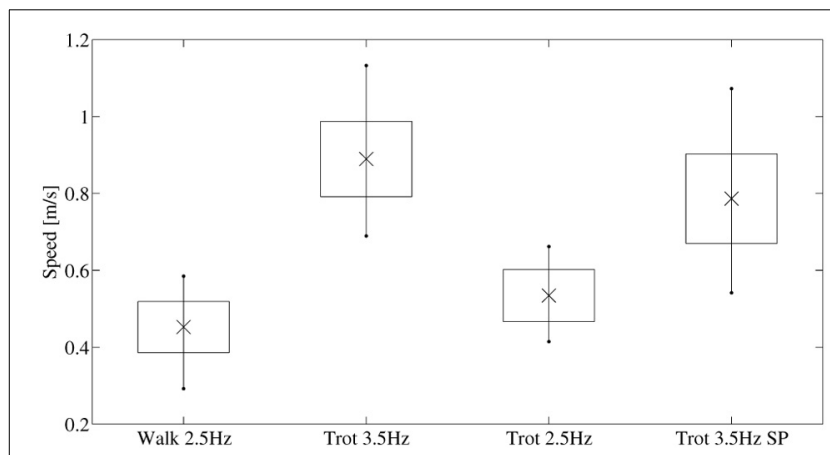

FIGURE 4 | Average speed values of the four experiments, sorted by gait type (walk, trot) and gait frequency $(\boldsymbol{f}=\mathbf{2 . 5}, \mathbf{3 . 5} \mathbf{~ H z}$ ). The $3.5 \mathrm{~Hz}$ trot applied a single-peak knee-trajectory (SP), the three remaining experiments applied double-peak knee-trajectories (DP). For the applied RC servo motor voltage of $12 \mathrm{~V}$, a trot gait speed of $0.9 \mathrm{~ms}^{-1}(\mathrm{FR}=1)$ is about the maximum average speed. of the variance of the kinematic joint-angle data, the first PC accounts for $58 \%$ of the variance, the second PC $40 \%$ of the variance. On-ground locomotion showed four PCs, accounting for $96 \%$ of the variance. The number of in-air PCs differed, compared to all other experiments (Figure 9D). The major change was a switch of the complexity of the knee-control signal, from double-peak to single-peak. This reduction was reflected in the in-air PC data, however, not in the on-ground PC data.

\subsection{COMBINED DATA}

In Figure 10, PCs of in-air leg movements and on-ground locomotion of all four experiments are depicted. This common pool of all collected joint-angle kinematics includes lateral sequence walk and trot gait data. The corresponding joint-angle plot was omitted, it basically covers the entire plot area. Three in-air PCs account for $97 \%$ of the variance, and four on-ground PCs account for $95 \%$ of the variance.

\section{DISCUSSION}

We presented results of locomotion patterns in-air and onground, for the two gait types walk and trot. The robot ran at average forward speeds between $v_{\mathrm{av}}=0.45 \mathrm{~ms}^{-1}$, more than 2 body lengths per second, and $v_{\mathrm{av}}=0.9 \mathrm{~ms}^{-1}$, around 4.5 body lengths
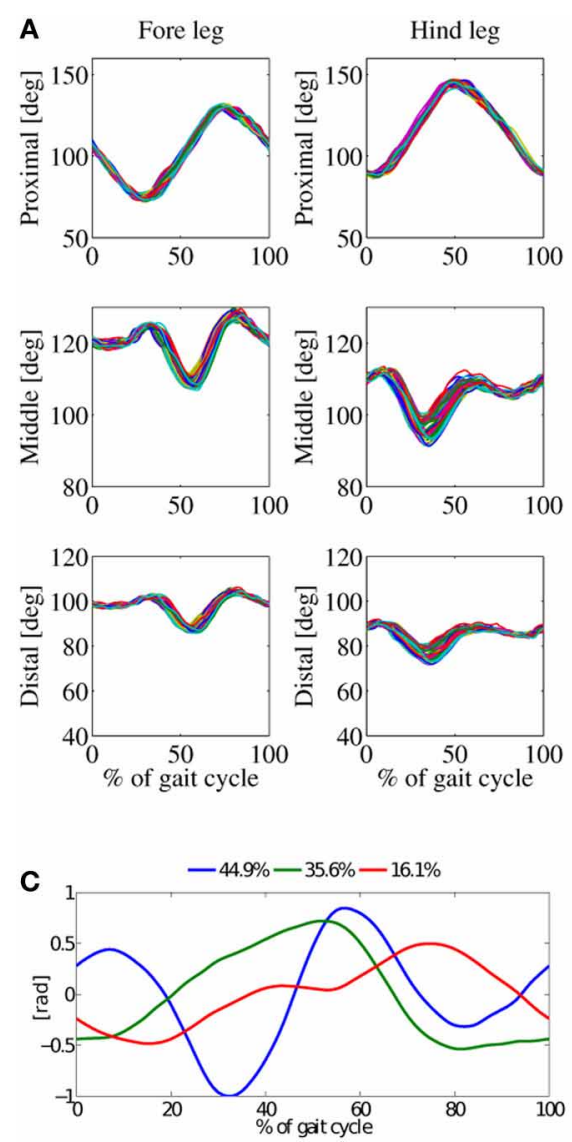

FIGURE 5 | Results from walk-gait experiments, at a locomotion frequency of $2.5 \mathrm{~Hz}$, with double-peak knee activation patterns. (A) Joint angles for in-air experiment, and (B) joint-angles for on-ground experiment.
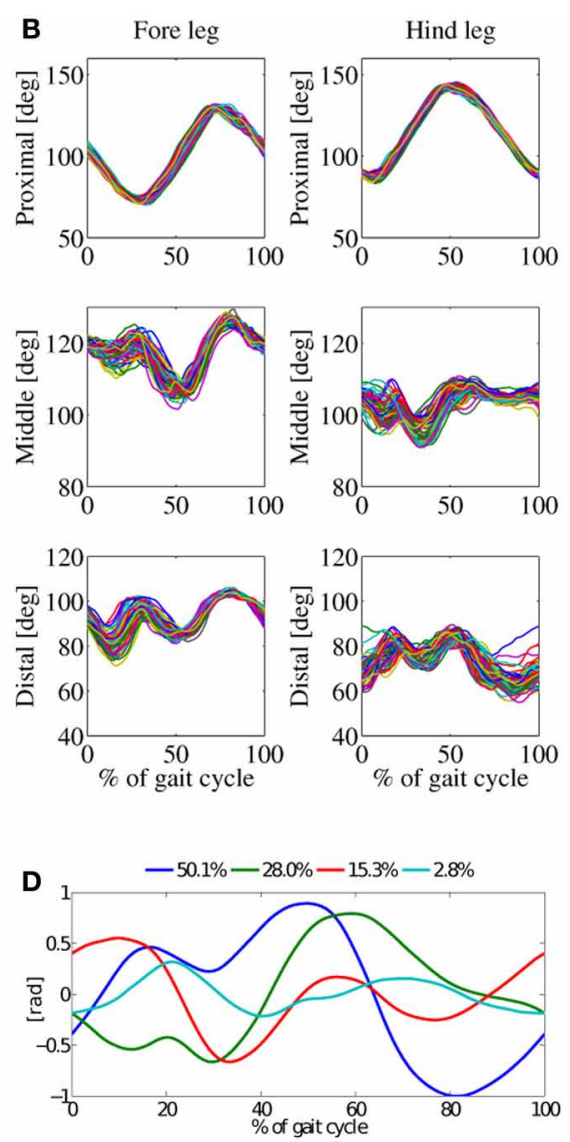

(C) Principal components (PC) for the in-air experiment, and (D) PCs for the on-ground experiment. Three basic patterns of the in-air experiment sum up to $97 \%$, the four basic pattern of the on-ground experiment to $96 \%$. 

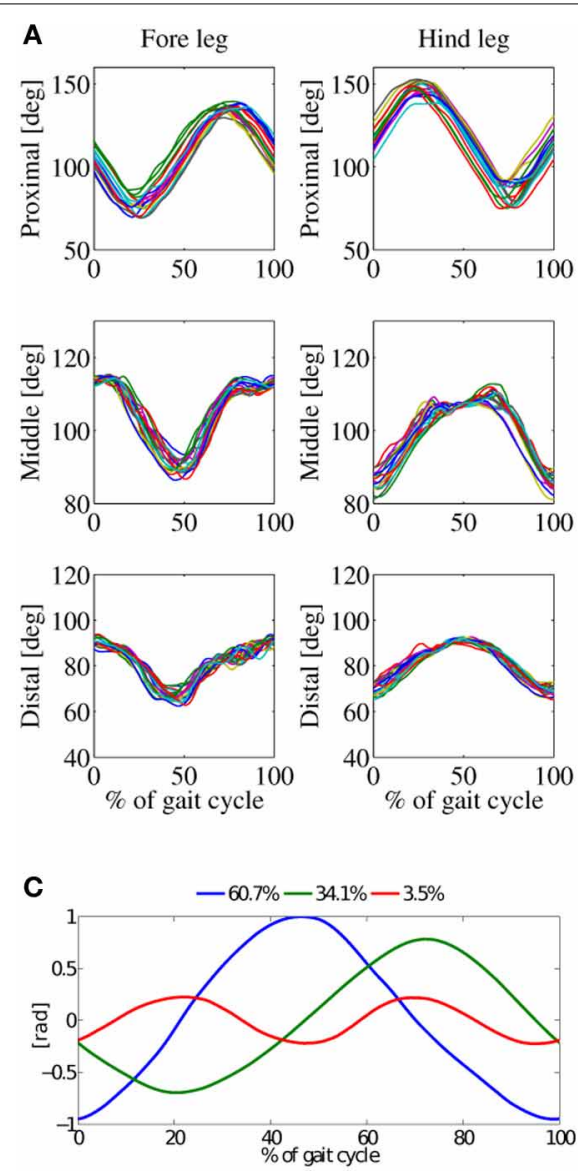

FIGURE 6 | Trot-gait experiments, locomotion frequency $3.5 \mathrm{~Hz}$, average robot speed was $0.9 \mathrm{~ms}^{-1}$, maximum speed was $1.1 \mathrm{~ms}^{-1}$, double-peak knee activation patterns. (A) Joint angles of in-air, and (B) joint-angles of
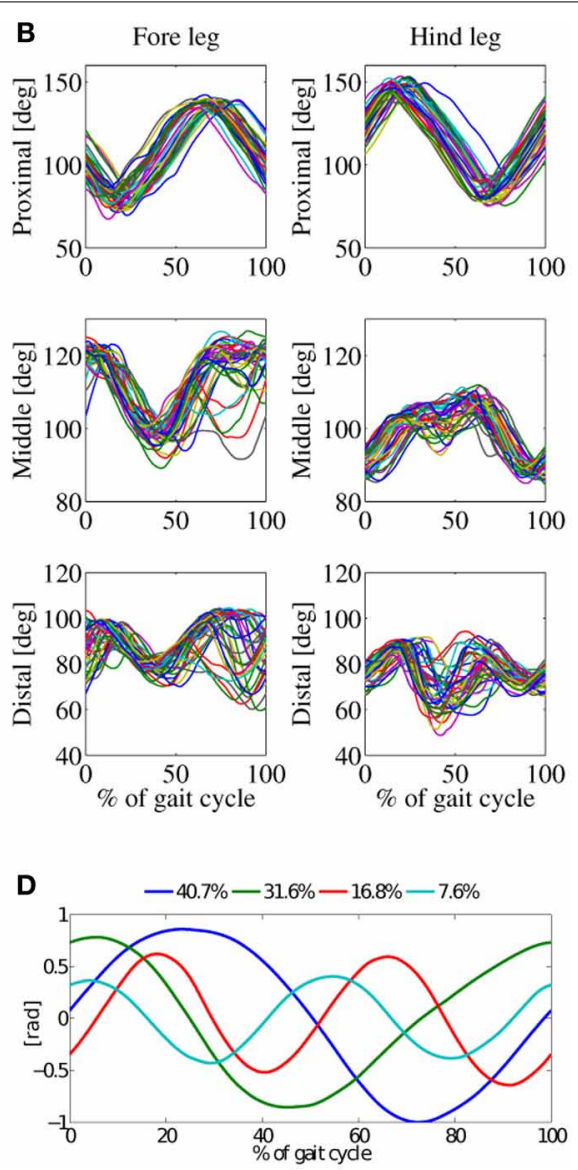

on-ground. (C) Principal components for the in-air experiment, and (D) PCs for the on-ground experiment. Three basic patterns of the in-air experiment sum up to $98 \%$, the four basic pattern of the on-ground experiment to $97 \%$. per second. All on-ground locomotion experiments showed four principal components. In-air experiments revealed either two PCs (single-peak knee controller) or three PCs (double-peak knee controller), accounting for at least $95 \%$ of the variance of the kinematic leg data.

Stable walk and trot gait pattern were derived by encoding joint control patterns as a set of coupled oscillators (CPG), and manually tuning CPG parameters. For all speeds and gaits, only one type of hip joint pattern was sufficient: a duty-factor distorted sine-wave position signal (Figure 3, top plot). For lower speed gaits, double-peak knee signals (Figure 3, mid figure) were necessary to produce stable gait patterns. For higher speed, both singlepeak (Figure 3, bottom plot) and double-peak signals produce stable gait patterns.

\subsection{IMPACT OF MECHANICAL ENTRAINMENT}

For all experiments mounting the robot on a stand and moving legs in-air, gaits with double-peak knee signals (DP-trot and DPwalk) showed three principal components accounting for at least 95\% of the variance. All experiments on-ground, independent from the robot speed, showed four basic patterns. These results support the first hypothesis (H1-1), derived from observations with animals; level-ground locomotion showed four to five basic components (Ivanenko et al., 2004; Dominici et al., 2011; Moro et al., 2013a,b).

Our in-air observations (hypothesis H2) coincide qualitatively with observations from human in-air stepping exhibiting simpler, harmonic leg kinematics (Ivanenko et al., 2002), essentially a lower observed kinematic complexity. However, we were unable to find quantitative descriptions of PCs for in-air stepping in animals with feed-forward-only motor control. Until otherwise reported, we consider the occurrence of either 2 or 3 PCs for the in-air patterns corresponding to 4 on-ground PCs as a weak indication for a similar mechanism in animals. Only similarly conducted animal experiments could potentially reveal evidential details on feed-forward motor control mechanisms in animals.

The interaction of the robot with its environment (i.e., ground contact) increased the kinematic complexity by at least one principal component. Qualitatively, this was externally observable through emerging robot body pitch and roll patterns.

For the single-peak knee actuation experiment $(\mathrm{SP}, 3.5 \mathrm{~Hz}$, trot) Cheetah-cub robot ran at high speed on-ground, in average $0.8 \mathrm{~ms}^{-1}$. Replaying the same CPG drive signals 

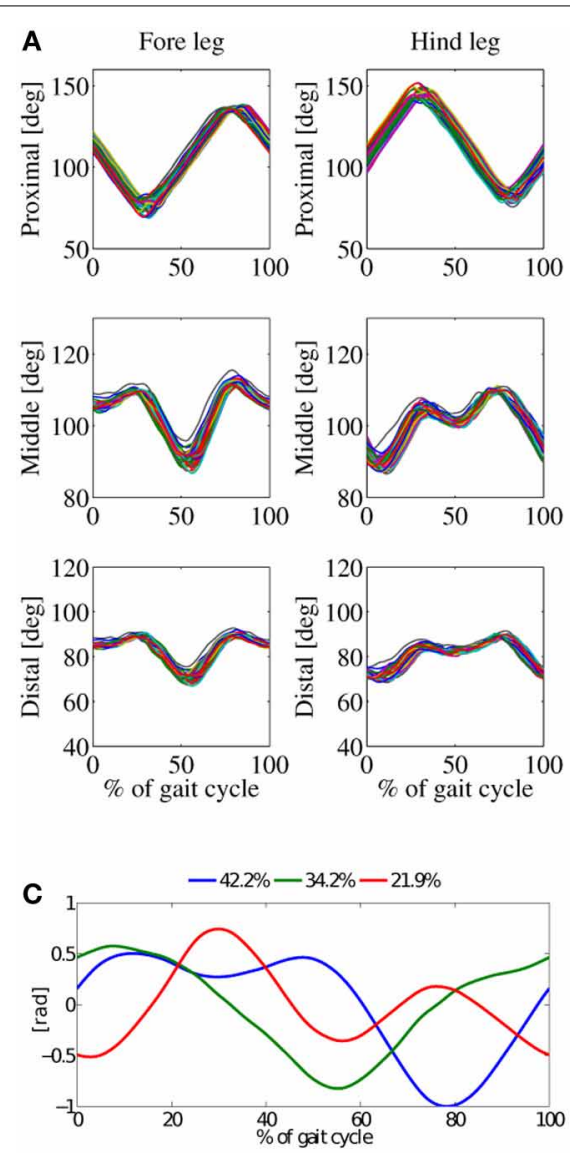

FIGURE 7 | Results from trot-gait experiments at a locomotion frequency $2.5 \mathrm{~Hz}$. Average robot speed was $0.55 \mathrm{~ms}^{-1}$, double-peak knee activation patterns. (A) Joint angles for in-air experiment, and (B) joint-angles for on-ground experiment. (C) Principal components
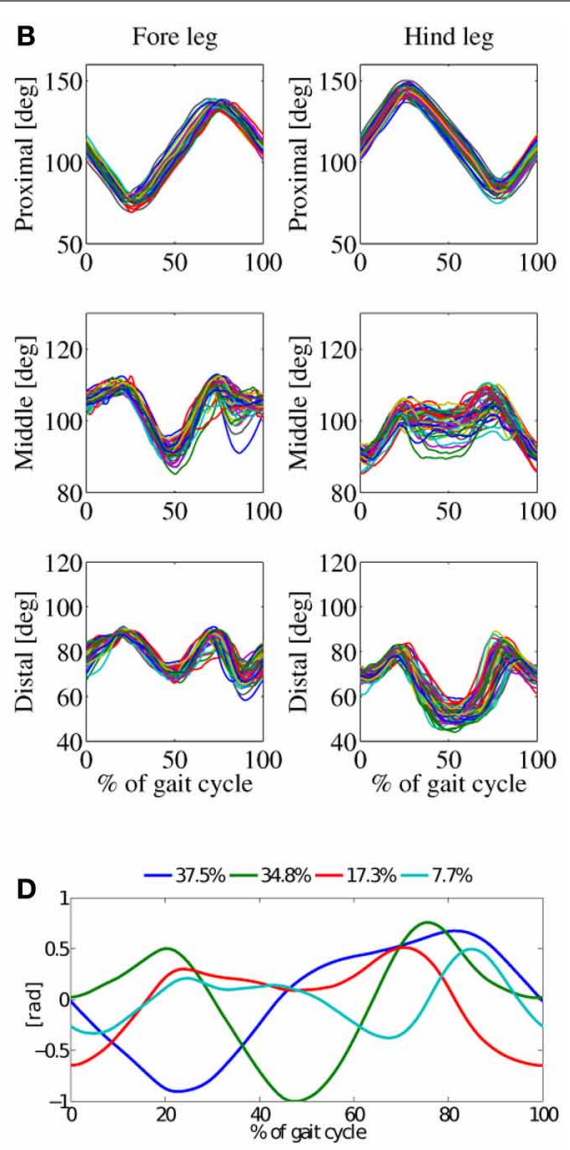

(PC) for the in-air experiment, and (D) PCs for the on-ground experiment. Three basic patterns of the in-air experiment sum up to $98 \%$, the four basic pattern of the on-ground experiment to $98 \%$. in-air showed two basic patterns (98\%, Figure 8C). We found stable on-ground SP-gait patterns only at higher robot speeds, from 0.55 to $1.1 \mathrm{~ms}^{-1}$. From this speed on, the robot's leg springs were sufficiently deflected by inertial forces and gravity, acting on the robot body, and enabled mechanical entrainment. Four basic patterns accounted for at least $95 \%$ of the variance of onground locomotion, for the SP-gait experiment. This result is not in accordance with the second part of hypothesis 1 (H1-2); a decreasing number of on-ground PCs was found at higher animal speeds for cockroaches (Koditschek et al., 2004). However, Cheetah-cub robot is unable to perform normalized speeds documented for these insects. The maximum robot speed recorded was 6.9 body lengths per second, or (Froude number of FR = 1.3 Spröwitz et al., 2013). It is possible that Cheetah-cub is not running fast enough to replicate similar results. Above results have potential implications for the general implementation of quadruped robot locomotion controllers: at level-ground running, the resulting patterns on-ground require not more than four basic components. This reduces the necessary complexity of the locomotion controller, also for other controller types than CPGs.

\subsection{CONTROL DIMENSION REDUCTION AND ROBOTIC GAIT GENERATION}

The CPG model used for the control of locomotion included more that 10 open parameters, tuned for each gait. We hypothesize that with a collected dataset of sufficient size, the extracted in-air PCs representing this dataset can be used to reconstruct a new controller. Hence, to generate a new gait, one could tune the in-air primitive-weights for different joints, instead of CPG control parameters. However, many additional experiments are required to prove this claim. If above weight-tuning would apply, one could also encode the extracted primitives into a Dynamical Movement Primitive (Ijspeert et al., 2003, 2013). This would provide smooth modulation of the output signals as well as feedback incorporating capabilities (Ajallooeian et al., 2013). Such a controller would allow for switching between different gaits, by a change of primitive weights.

At present, we found no quantitative data available to compare our results with other legged robotic platforms, to observe the effect of mechanical entrainment of feed-forward controlled, legged robots and their kinematic motion primitives. Platforms other than Cheetah-cub robot exist, locomoting with 

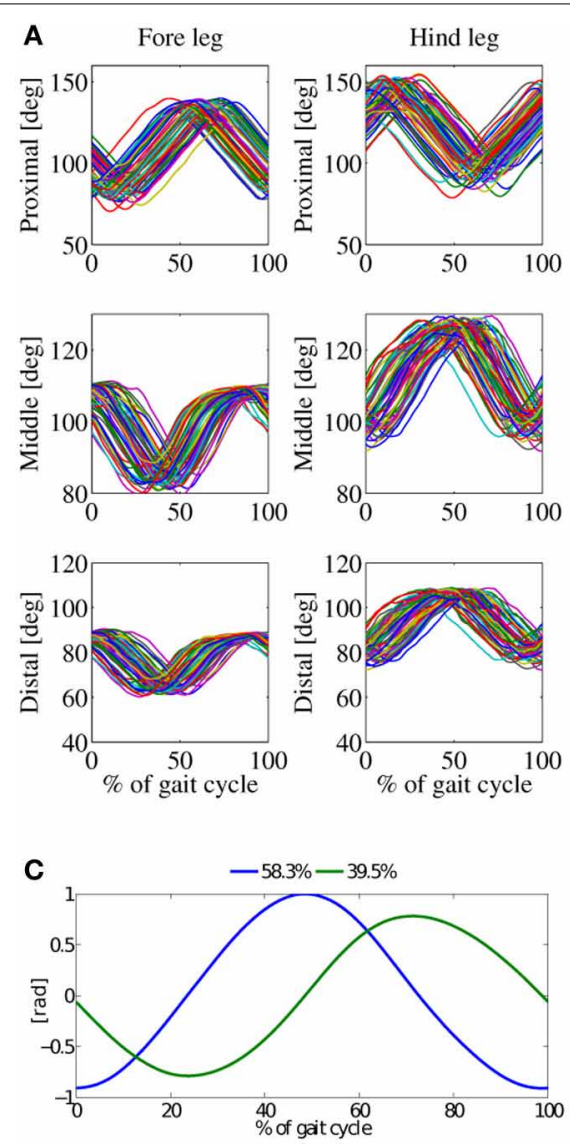

FIGURE 8 | Results from the trot-gait experiments, at a locomotion frequency $3.5 \mathbf{H z}$. The average robot speed was $0.8 \mathrm{~ms}^{-1}$, single-peak knee activation patterns. (A) Joint angles for in-air experiment, and (B) joint-angles for on-ground experiment.
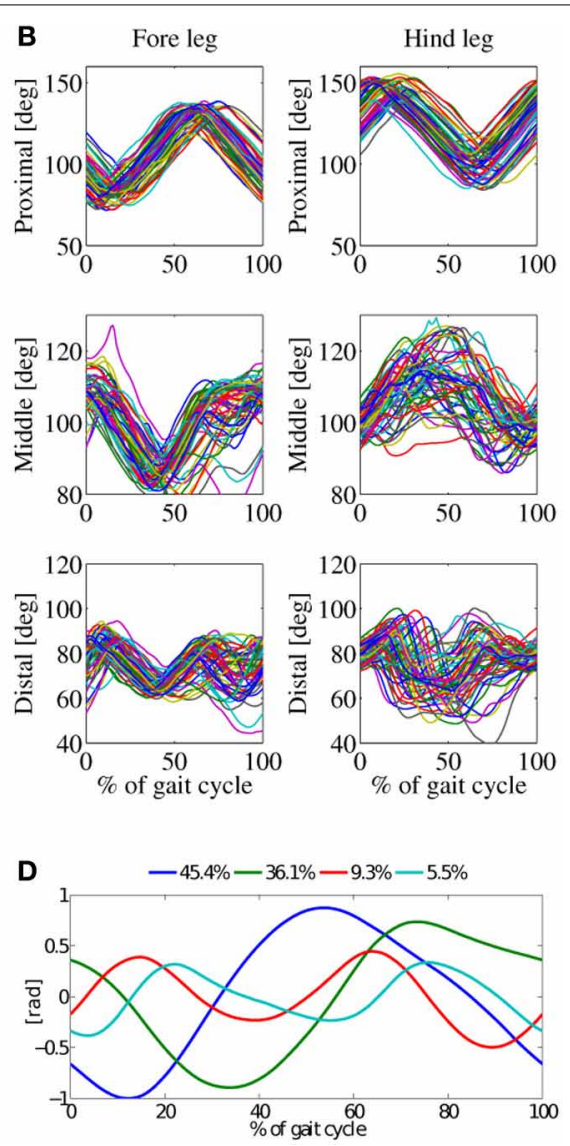

(C) Principal components (PC) for the in-air experiment, and (D) PCs for the on-ground experiment. Two basic patterns of the in-air experiment sum up to $98 \%$, the four basic pattern of the on-ground experiment to $96 \%$. dynamical, feed-forward controlled, self-stabilizing gaits and similar leg mechanics. Bobcat-robot for example is a small bounding robot and it can reach dynamical, full flight phases in-between touch-downs. It is equipped with a Cheetah-cublike feed-forward controller, in-series elastic segmented legs, and an actuated spine (Khoramshahi et al., 2013). However, no data on the complexity of its kinematic on-ground PCs is available. Typically, motor controller designs for legged robots feature explicit feedback loops (Kimura et al., 2007; Buchli et al., 2009).

Mechanical compliance in legged robotic design has been introduced very early; Raibert's robots featured in-series elasticity (air springs, Raibert et al., 1984). Raibert reported a closed-loop controller with explicit, though simple and linear feedback. We are unaware on how much Raibert's machines could have been controlled in a feed-forward manner. We experienced that sufficiently complex feed-forward CPG signals (two to three PCs, speed dependent), a segmented leg design, and in-series and in-parallel leg compliance were the necessary ingredients for a simple, yet self-stable, dynamically legged quadruped robot system. Cheetah-cub and other robots (Iida and Pfeifer, 2004; Khoramshahi et al., 2013) are indicators that a larger design pool for mechanically entrained, dynamical robots exists.

As for the robot's range of leg compliance; Cheetah-cub features a slightly lower leg stiffness than observed in animals of the same weight and leg length. It would be an interesting future experiment to incrementally alter the leg stiffness, up to a level where the leg has no compliance. This would also require a way to alter the feed-forward control patterns in a systematic way, to ensure comparability between resulting gaits.

\subsection{RELEVANCE TO BIOLOGICAL SYSTEMS}

Cheetah-cub is a bio-inspired robot designed and motion controlled according to bio-inspired blueprints. Therefore, it presents a strong abstraction. We replicated observed blueprints from functional anatomy (pantograph leg, in-series and in-parallel compliance, clutch mechanism) and control (CPGs and locomotion parameters: duty factor, leg length, leg angle, amplitude, and frequency). The applied feed-forward controller produced position-control motor signals. Swing-leg dynamics are different to that of an animal, because its mechanical spring force cannot be manipulated in an online fashion. The robot's distal compliance acts passively and in-series, whereas quadruped animals are 


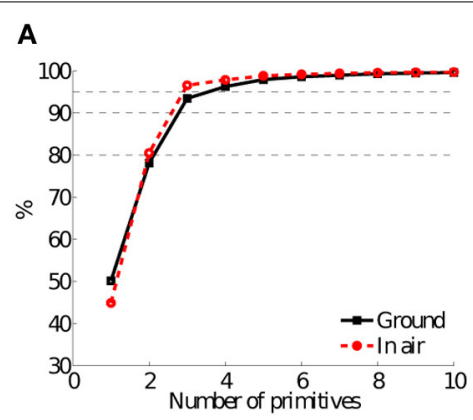

C

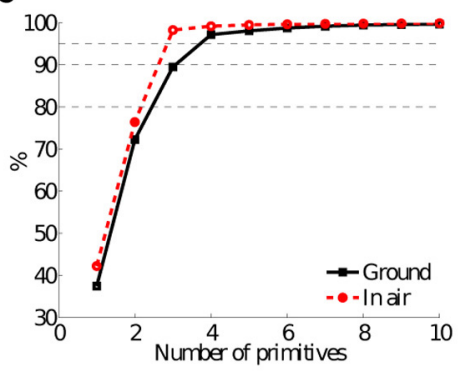

FIGURE 9 | The percent variance accounting for the first 10 primitives, as a function of the number of primitives, for in-air stepping (red, dashed lines) and on-ground (black, solid lines) locomotion patterns. Horizontal, black, dashed lines indicate $80 \%$, $90 \%$, and $95 \%$ of the variance. In this article, we used a $95 \%$ of the variance interval (top, dashed, black, horizontal line). This results in between 2 and 4 primitives to account for $\geq 95 \%$ of the variance. The single peak (SP) trot gait at $3.5 \mathrm{~Hz}$ (D) showed the largest change from
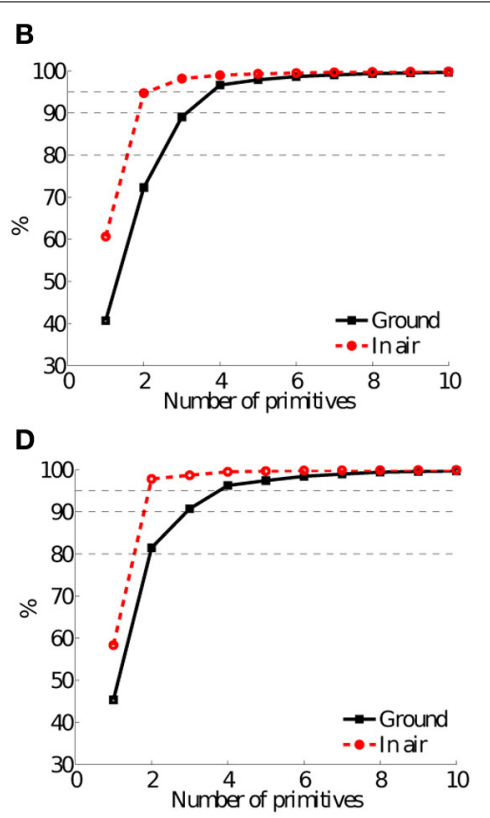

in-air stepping to on-ground locomotion: 2 primitives accounted for more than $95 \%$ of the variance of in-air stepping data, and 4 primitives accounted for the same variance threshold, for on-ground locomotion. In the three other (A-C) experimental setups 3 primitives accounted for at least $95 \%$ of the variance of kinematic in-air stepping data, and 4 primitives accounted for the same threshold of on-ground locomotion. (A) Walk, $2.5 \mathrm{~Hz}$, DP. (B) Trot, $3.5 \mathrm{~Hz}$, DP. (C) Trot, $2.5 \mathrm{~Hz}$, DP. (D) Trot, $3.5 \mathrm{~Hz}, \mathrm{SP}$.
FIGURE 10 | PCs extracted from joint-angle trajectories of all four experiments, i.e., walk and trot, single (SP) and double-peak (DP) knee actuation, and 2.5 and $3.5 \mathrm{~Hz}$ locomotion frequency. (A) PCs from
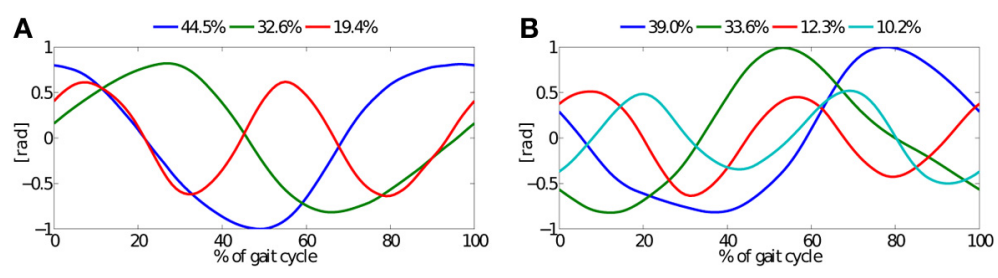

in-air experiment, and (B) PCs from on-ground experiment. Three basic patterns account for $97 \%$ of the variance during in-air stepping, $95 \%$ for on-ground locomotion. able to adjust ankle stiffness. Cheetah-cub does not feature an antagonist actuator producing a foot-joint stiffness profile.

Studies on legged locomotion in biology indicate that 4-5 principal components account for a large part of kinematic data variance, both for vertebrates and invertebrates (Ivanenko et al., 2004; Holmes et al., 2006; Dominici et al., 2011; Moro et al., 2013a,b). This is remarkable, considering the range of leg lengths, body sizes and weights, and differences in leg design and actuation strategies. In our study, four PCs accounted for $95 \%$ of the variance of all level-ground experimental data, including walk and trot gait data. The corresponding locomotion controller (CPG in feed-forward mode) was programmed with changing complexity. Depending on the range of robot speed either 3 or 2 PCs accounted for the variance of the feed-forward controller data (slower and faster in-air patterns, respectively). Perturbation experiments with running birds (guinea fowl) indicate that bipedal running is controlled through a combination of feed-forward control and additional, reflex-based actuator changes (cf. Daley et al., 2006, 2009). For future robotic legged experiments it will become interesting to observe and quantify the effect of explicit, possibly reflex-based feedback. Adult human locomotion patterns showed more pronounced basic gait patterns, compared to those of toddlers (cf. Dominici et al., 2011). These patterns were also phase-locked at important gait events. It is also unclear if and how a faster running robotic system would reduce the number of observable principal components 
with increasing robot speed, similar to the findings of Koditschek et al. (2004).

\section{CONCLUSION}

In this study we reported on the interplay between a modular, feed-forward locomotion controller, and the mechanical entrainment of a quadruped, self-stably walking and trotting compliant legged robot. We measured the complexity of the feed-forward controller, and the complexity of the resulting leg kinematics through the number of basic patterns accounting for a certain variance of kinematic data from in-air leg motions and on-ground locomotion, respectively. We implemented lateral sequence walk and trot gaits, and applied two different leg length control strategies. We found that the number of basic patterns from on-ground locomotion data matched those reported for animals; four basic patterns accounted for $\geq 95 \%$ of the variance. Three basic patterns accounted for $\geq 95 \%$ of the variance in lateral sequence walk and slower trot in-air experimental kinematic data, and two basic patterns accounted for faster trotting. Because patterns were sent in a feed-forward manner, the measured complexity of in-air kinematic data represents the complexity of the feed-forward controller. This shows that already a simple, modular rhythm generator is sufficient for level-ground, feedforward legged quadruped locomotion, for two different gaits walk and trot. It also shows that passive mechanical compliance enables an increase of kinematic complexity, leading to dynamic and self-stabilizing walk and trot locomotion. In the case of our quadruped legged robot, the complexity of the kinematic data increased at ground contact, through mechanical entrainment between the feed-forward controller and compliant, bio-inspired robot hardware. Here, the bio-inspired leg design supported the emergence of additional on-ground basic primitives, e.g., through passive leg compliance and leg segmentation. Animals show a much wider range of tools to adapt and modulate dynamic legged locomotion. Similar results between presented robot experiments and experiments with animals at level-ground locomotion indicate that modular, feed-forward, rhythmic pattern-based motor control in combination with compliant hardware are important components of animal neuro-control and bio-mechanics.

\section{ACKNOWLEDGMENTS}

The research leading to these results has received funding from the European Community's Seventh Framework Programme FP7/2007-2013-Challenge 2-Cognitive Systems, Interaction, Robotics-under grant agreement No. 248311 (AMARSi), and from the Swiss National Science Foundation through the National Centre of Competence in Research Robotics.

\section{REFERENCES}

Ajallooeian, M., Gay, S., Tuleu, A., Spröwitz, A., and Ijspeert, A. J. (2013). "Modular control of limit cycle locomotion over unperceived rough terrain," in Intelligent Robots and Systems (IROS), 2013 IEEE/RSJ International Conference on (Tokyo: IEEE), 3390-3397. doi: 10.1109/IROS.2013.6696839

Alexander, R. M. (1977). Storage of elastic strain energy in muscle and other tissues. Nature 265, 114-117. doi: 10.1038/265114a0

Alexander, R. M. (1984). Elastic energy stores in running vertebrates. Am. Zool. 24, 85-94.

Alexander, R. M. (1990). Three uses for springs in legged locomotion. Int. J. Robot. Res. 9, 53-61. doi: 10.1177/027836499000900205
Alexander, R. M. (1991). Energy-saving mechanisms in walking and running. J. Exp. Biol. 160, 55-69.

Barré, A., and Armand, S. (2014). Biomechanical ToolKit: Open-source framework to visualize and process biomechanical data. Comput. Methods Programs Biomed. doi: 10.1016/j.cmpb.2014.01.012. [Epub ahead of print].

Berthouze, L., and Lungarella, M. (2004). Motor skill acquisition under environmental perturbations: on the necessity of alternate freezing and freeing of degrees of freedom. Adapt. Behav. 12, 47-64. doi: 10.1177/1059712304012 00104

Biewener, A. A. (1998). Muscle-tendon stresses and elastic energy storage during locomotion in the horse. Comp. Biochem. Physiol. B Biochem. Mol. Biol. 120, 73-87. doi: 10.1016/S0305-0491(98)00024-8

Biewener, A. A., and Blickhan, R. (1988). Kangaroo rat locomotion: design for elastic energy storage or acceleration? J. Exp. Biol. 140, 243-255.

Bizzi, E., Cheung, V., d'Avella, A., Saltiel, P., and Tresch, M. (2008). Combining modules for movement. Brain Res. Rev. 57, 125-133. doi: 10.1016/j.brainresrev.2007.08.004

Blickhan, R. (1989). The spring-mass model for running and hopping. J. Biomech. 22, 1217-1227. doi: 10.1016/0021-9290(89)90224-8

Buchli, J., Kalakrishnan, M., Mistry, M., Pastor, P., and Schaal, S. (2009). "Compliant quadruped locomotion over rough terrain," in Proceedings of the 2009 IEEE/RSJ International Conference on Intelligent Robots and Systems (St. Louis, MO: IEEE Press), 814-820. doi: 10.1109/IROS.2009.5354681

Daley, M. A., Usherwood, J. R., Felix, G., and Biewener, A. A. (2006). Running over rough terrain: guinea fowl maintain dynamic stability despite a large unexpected change in substrate height. J. Exp. Biol. 209, 171-187. doi: 10.1242/jeb.01986

Daley, M. A., Voloshina, A. S., and Biewener, A. A. (2009). The role of intrinsic muscle mechanics in the neuromuscular control of stable running in the guinea fowl. J. Physiol. 587, 2693-2707. doi: 10.1113/jphysiol.2009.171017

Daou, I., Tuttle, A. H., Longo, G., Wieskopf, J. S., Bonin, R. P., Ase, A. R., et al. (2013). Remote optogenetic activation and sensitization of pain pathways in freely moving mice. J. Neurosci. 33, 18631-18640. doi: 10.1523/JNEUROSCI.2424-13.2013

Delcomyn, F. (1980). Neural basis of rhythmic behavior in animals. Science 210, 492-498. doi: 10.1126/science.7423199

Dominici, N., Ivanenko, Y. P., Cappellini, G., d'Avella, A., Mondì, V., Cicchese, M., et al. (2011). Locomotor primitives in newborn babies and their development. Science 334, 997-999. doi: 10.1126/science.1210617

Farley, C., Glasheen, J., and McMahon, T. (1993). Running springs: speed and animal size. J. Exp. Biol. 185, 71-86.

Fischer, M., and Blickhan, R. (2006). The tri-segmented limbs of therian mammals: kinematics, dynamics, and self-stabilization-a review. J. Exp. Zool. A Comp. Exp. Biol. 305, 935-952. doi: 10.1002/jez.a.333

Fod, A., Mataric, M. J., and Jenkins, O. C. (2002). Automated derivation of primitives for movement classification. Auton. Robot. 12, 39-54. doi: 10.1023/A:1013254724861

Fritsch, F. N., and Carlson, R. E. (1980). Monotone piecewise cubic interpolation. SIAM J. Numer. Anal. 17, 238-246. doi: 10.1137/0717021

Fukuoka, Y., Kimura, H., and Cohen, A. H. (2003). Adaptive dynamic walking of a quadruped robot on irregular terrain based on biological concepts. Int. J. Robot. Res. 22, 187-202. doi: 10.1177/0278364903022003004

Full, R., and Koditschek, D. (1999). Templates and anchors: neuromechanical hypotheses of legged locomotion on land. J. Exp. Biol. 202, 3325-3332.

Gregersen, C. S., Silverton, N. A., and Carrier, D. R. (1998). External work and potential for elastic storage at the limb joints of running dogs. J. Exp. Biol. 201, 3197-3210.

Grillner, P. S., and Zangger, P. (1979). On the central generation of locomotion in the low spinal cat. Exp. Brain Res. 34, 241-261. doi: 10.1007/BF00235671

Grillner, S. (1985). "Neural control of vertebrate locomotion — central mechanisms and reflex interaction with special reference to the cat," in Feedback and Motor Control in Invertebrates and Vertebrates, eds W. J. P. Barnes and M. H. Gladden (London: Croom Helm), 35-56.

Hägglund, M., Dougherty, K. J., Borgius, L., Itohara, S., Iwasato, T., and Kiehn, O. (2013). Optogenetic dissection reveals multiple rhythmogenic modules underlying locomotion. Proc. Natl. Acad. Sci. U.S.A. 110, 11589-11594. doi: $10.1073 /$ pnas. 1304365110

Havoutis, I., Semini, C., Buchli, J., and Caldwell, D. (2013). “Quadrupedal trotting with active compliance," in 2013 IEEE International Conference on Mechatronics (Vicenza), 610-616. doi: 10.1109/ICMECH.2013.6519112 
Hildebrand, M. (1989). The quadrupedal gaits of vertebrates. Bioscience 39, 766-775. doi: $10.2307 / 1311182$

Holmes, P., Full, R. J., Koditschek, D., and Guckenheimer, J. (2006). The dynamics of legged locomotion: models, analyses, and challenges. SIAM Rev. 48, 207-304. doi: $10.1137 / S 0036144504445133$

Iida, F., and Pfeifer, R. (2004). “"Cheap" rapid locomotion of a quadruped robot: self-stabilization of bounding gait," in Proceedings of the 8th International Conference on Intelligent Autonomous Systems (IAS-8), ed F. Groen (Amsterdam: IOS Press), 642-649.

Ijspeert, A. J. (2008). Central pattern generators for locomotion control in animals and robots: a review. Neural Netw. 21, 642-653. doi: 10.1016/j.neunet.2008.03.014

Ijspeert, A. J., Crespi, A., Ryczko, D., and Cabelguen, J.-M. (2007). From swimming to walking with a salamander robot driven by a spinal cord model. Science 315, 1416-1420. doi: 10.1126/science.1138353

Ijspeert, A. J., Nakanishi, J., Hoffmann, H., Pastor, P., and Schaal, S. (2013). Dynamical movement primitives: learning attractor models for motor behaviors. Neural Comput. 25, 328-373. doi: 10.1162/NECO_a_00393

Ijspeert, A. J., Nakanishi, J., and Schaal, S. (2003). "Learning attractor landscapes for learning motor primitives," in Advances in Neural Information Processing Systems 15 (NIPS2002), eds S. Becker, S. Thrun, and K. Obermayer (Cambridge, MA: MIT Press), 1547-1554.

Inman, V. T., Ralston, H. J., De C.M. Saunders, J. B., Bertram Feinstein, M. B., and Wright Jr., E. W. (1952). Relation of human electromyogram to muscular tension. Electroencephalogr. Clin. Neurophysiol. 4, 187-194. doi: 10.1016/00134694(52)90008-4

Ivanenko, Y. P., Grasso, R., Macellari, V., and Lacquaniti, F. (2002). Control of foot trajectory in human locomotion: role of ground contact forces in simulated reduced gravity. J. Neurophysiol. 87, 3070-3089.

Ivanenko, Y. P., Poppele, R. E., and Lacquaniti, F. (2004). Five basic muscle activation patterns account for muscle activity during human locomotion. J. Physiol. 556, 267-282. doi: 10.1113/jphysiol.2003.057174

Jolliffe, I. T. (2002). Principal Component Analysis. 2nd Edn. New York, NY: Springer.

Khoramshahi, M., Spröwitz, A., Tuleu, A., Ahmadabadi, M., and Ijspeert, A. (2013). "Benefits of an active spine supported bounding locomotion with a small compliant quadruped robot," in Proceedings of 2013 IEEE International Conference on Robots and Automation (Karlsruhe). doi: 10.1109/ICRA.2013.6631041

Kimura, H., Fukuoka, Y., and Cohen, A. (2007). Adaptive dynamic walking of a quadruped robot on natural ground based on biological concepts. Int. J. Robot. Res. 26, 475-490. doi: 10.1177/0278364907078089

Koditschek, D., Full, R. J., and Buehler, M. (2004). Mechanical aspects of legged locomotion control. Arthropod Struct. Dev. 33, 251-272. doi: 10.1016/j.asd.2004.06.003

Krzanowski, W. (1988). Principles of Multivariate Analysis; A Users Perspective. Oxford statistical science series, Vol. 3. Oxford, NY: Oxford University Press.

Labeit, S., and Kolmerer, B. (1995). Titins: giant proteins in charge of muscle ultrastructure and elasticity. Science 270, 293-296. doi: 10.1126/science.270.5234.293

Lichtwark, G., Bougoulias, K., and Wilson, A. (2007). Muscle fascicle and series elastic element length changes along the length of the human gastrocnemius during walking and running. J. Biomech. 40, 157-164. doi: 10.1016/j.jbiomech.2005.10.035

Lungarella, M., and Berthouze, L. (2002). On the interplay between morphological, neural, and environmental dynamics: a robotic case study. Adapt. Behav. 10, 223-241. doi: 10.1177/1059712302010003005

MATLAB. (2009). Version 7.09.0 (R2009b). Natick, MA: The MathWorks Inc.

Meyer, F., Spröwitz, A., and Berthouze, L. (2006). Passive compliance for a RC servo-controlled bouncing robot. Adv. Robot. 20, 953-961. doi: $10.1163 / 156855306777951429$

Moro, F. L., Spröwitz, A., Tuleu, A., Vespignani, M., Tsagakiris, N. G., Ijspeert, A., et al. (2013a). Horse-like walking, trotting, and galloping derived from kinematic motion primitives (kmps) and their application to walk/trot transitions in a compliant quadruped robot. Biol. Cybern. 107, 309-320. doi: 10.1007/s00422-013-0551-9

Moro, F. L., Tsagarakis, N. G., and Caldwell, D. G. (2013b). Walking in the resonance with the COMAN robot with trajectories based on human kinematic motion primitives (kMPs). Auton. Robot. 36, 331-347. doi: 10.1007/s10514-0139357-9

Naturalpoint, Inc. (2011). Optitrack s250e. Corvallis, OR: Naturalpoint, Inc.
Raibert, M., Chepponis, M., and Brown, H. (1986). Running on four legs as though they were one. Robot. Automat. 2, 70-82. doi: 10.1109/JRA.1986.1087044

Raibert, M. H. (1990). Trotting, pacing and bounding by a quadruped robot. J. Biomech. 23, 79-81. doi: 10.1016/0021-9290(90)90043-3

Raibert, M. H., Brown, H. B., and Chepponis, M. (1984). Experiments in balance with a 3D one-legged hopping machine. Int. J. Robot. Res. 3, 75-92. doi: $10.1177 / 027836498400300207$

Righetti, L., and Ijspeert, A. (2008). "Pattern generators with sensory feedback for the control of quadruped locomotion," in IEEE International Conference on Robotics and Automation, 2008. ICRA 2008 (Pasadena, CA), 819-824. doi: 10.1109/ROBOT.2008.4543306

Sato, T., Kano, T., and Ishiguro, A. (2011). On the applicability of the decentralized control mechanism extracted from the true slime mold: a robotic case study with a serpentine robot. Bioinspir. Biomim. 6:026006. doi: 10.1088/17483182/6/2/026006

Seyfarth, A., Geyer, H., Guenther, M., and Blickhan, R. (2002). A movement criterion for running. J. Biomech. 35, 649-655. doi: 10.1016/S0021-9290(01) 00245-7

Spröwitz, A., Moeckel, R., Maye, J., and Ijspeert, A. J. (2008). Learning to move in modular robots using central pattern generators and online optimization. Int. J. Robot. Res. 27, 423-443. doi: 10.1177/0278364907088401

Spröwitz, A., Tuleu, A., Vespignani, M., Ajallooeian, M., Badri, E., and Ijspeert, A. J. (2013). Towards dynamic trot gait locomotion-design, control, and experiments with cheetah-cub, a compliant quadruped robot. Int. J. Rob. Res. 32, 932-950. doi: 10.1177/0278364913489205

Strang, G. (2003). Introduction to Linear Algebra. Wellesley, MA: Wellesley Cambridge Press.

Umedachi, T., Takeda, K., Nakagaki, T., Kobayashi, R., and Ishiguro, A. (2010). Fully decentralized control of a soft-bodied robot inspired by true slime mold. Biol. Cybern. 102, 261-269. doi: 10.1007/s00422-010-0367-9

Valenzuela, A., and Kim, S. (2012). "Optimally scaled hip-force planning: a control approach for quadrupedal running," in 2012 IEEE International Conference on Robotics and Automation (ICRA) (Saint Paul, MN), 1901-1907. doi: 10.1109/ICRA.2012.6225251

Webb, B. (2001). Can robots make good models of biological behaviour? Behav. Brain Sci. 24, 1033-1050. doi: 10.1017/S0140525X01550128

Wilson, A. M., Watson, J. C., and Lichtwark, G. A. (2003). Biomechanics: a catapult action for rapid limb protraction. Nature 421, 35-36. doi: 10.1038/421035a

Winter, D. A. (1983). Moments of force and mechanical power in jogging. J. Biomech. 16, 91-97. doi: 10.1016/0021-9290(83)90050-7

Witte, H., Hackert, R., Ilg, W., Biltzinger, J., Schillinger, N., Biedermann, F., et al. (2003). "Quadrupedal mammals as paragons for walking machines," in Proceedings of AMAM - Adaptive Motion in Animals and Machines (Kyoto), TuA-II-2.1-TuA-II-2.4.

Witte, H., Rao, J., Lesch, C., Recknagel, S., and Preuschoft, H. (1994). Titin: a key towards an understanding of the elastic mechanisms in animal locomotion. Proc. EMC Cell Motil. Cell Res. 16, 10.

Witte, H., Recknagel, S., Rao, J. G., Wuethrich, M., and Lesch, C. (1997). Is elastic energy storage of quantitative relevance for the functional morphology of the human locomotor apparatus. Acta Anat. 158, 106-111. doi: 10.1159/000147919

Zhou, X., and Bi, S. (2012). A survey of bio-inspired compliant legged robot designs. Bioinspir. Biomim. 7:041001. doi: 10.1088/1748-3182/7/4/041001

Conflict of Interest Statement: The authors declare that the research was conducted in the absence of any commercial or financial relationships that could be construed as a potential conflict of interest.

Received: 30 September 2013; accepted: 20 February 2014; published online: 07 March 2014.

Citation: Spröwitz AT, Ajallooeian M, Tuleu A and Ijspeert AJ (2014) Kinematic primitives for walking and trotting gaits of a quadruped robot with compliant legs. Front. Comput. Neurosci. 8:27. doi: 10.3389/fncom.2014.00027

This article was submitted to the journal Frontiers in Computational Neuroscience. Copyright $\odot 2014$ Spröwitz, Ajallooeian, Tuleu and Ijspeert. This is an open-access article distributed under the terms of the Creative Commons Attribution License (CC BY). The use, distribution or reproduction in other forums is permitted, provided the original author(s) or licensor are credited and that the original publication in this journal is cited, in accordance with accepted academic practice. No use, distribution or reproduction is permitted which does not comply with these terms. 